\title{
Nonsingular recursion formulas for third-body perturbations in mean vectorial elements
}

\author{
M. Lara ${ }^{1,3}$, A. J. Rosengren ${ }^{2}$, and E. Fantino ${ }^{3}$ \\ ${ }^{1}$ Scientific Computing Group, University of La Rioja, Calle Madre de Dios 53, 26006 Logroño, Spain \\ e-mail: martin.lara@unirioja.es \\ 2 Department of Aerospace \& Mechanical Engineering, The University of Arizona, 1130 N. Mountain Ave., PO Box 210119, Tucson, \\ AZ, USA \\ e-mail: ajrosengren@email.arizona.edu \\ 3 Department of Aerospace Engineering, Khalifa University of Science and Technology, PO Box 127788, Abu Dhabi, UAE \\ e-mail: elena.fantino@ku.ac.ae
}

Received 13 November 2019 / Accepted 3 January 2020

\begin{abstract}
The description of the long-term dynamics of highly elliptic orbits under third-body perturbations may require an expansion of the disturbing function in series of the semi-major axes ratio up to higher orders. To avoid dealing with long series in trigonometric functions, we refer the motion to the apsidal frame and efficiently remove the short-period effects of this expansion in vectorial form up to an arbitrary order. We then provide the variation equations of the two fundamental vectors of the Keplerian motion by analogous vectorial recurrences, which are free from singularities and take a compact form useful for the numerical propagation of the flow in mean elements.
\end{abstract}

Key words. celestial mechanics

\section{Introduction}

Perturbed Keplerian motion is a multiscale problem, in which the orbital elements evolve slowly when compared to the change with time of the ephemeris, whose fast evolution is determined by the rate of variation of the mean anomaly. Usual integration schemes that look for the separation of fast and slow frequencies of the motion may be superior to the simpler integration with the Cowell method, in which the Newtonian acceleration is directly integrated in rectangular coordinates, and formulations based on Hansen's ideal frame concept (Hansen 1857) closely approach this decoupling (see Lara 2017, and references therein). Moreover, the use of symplectic integrators is widely adopted in the case of Hamiltonian perturbation problems (Laskar \& Robutel 2001; Blanes et al. 2013).

On the other hand, the effective decoupling of short- and long-period effects is achieved with perturbation methods. After removing short-period effects in an averaging process, the long-term behavior of the (mean) orbital elements is efficiently integrated from the equations of variation of parameters. The parameters, which are constant in the pure Keplerian motion, can take different representations, such as classical Keplerian elements or nonsingular variables (see Hintz 2008 for a survey), but are always related to the two fundamental vectors of the orbital motion: the angular momentum vector, which determines the orientation of the orbital plane, and the eccentricity vector, which determines the shape and orientation of the reference ellipse in that plane. This last is a nondimensional version of the classical Laplace vector, which has dimensions of the gravitational parameter, or of the Runge-Lenz vector, which has dimensions of angular momentum. While vectorial formulations normally introduce redundancy by increasing the dimension of the differential system, they commonly admit a compact and symmetric formulation of the right side of the variation equations that renders faster evaluation (Allan 1962; Musen 1963; Roy \& Moran $1973)^{1}$, and may even disclose integrability (Deprit 1984; see also Mignard \& Henon 1984; Richter \& Keller 1995).

The vectorial formulation has been shown to be useful in the case of third-body perturbations (Breiter \& Ratajczak 2005; Correia et al. 2011; Katz et al. 2011). In particular, it may be an efficient alternative to classical formulations when the orbits are highly elliptic, which is a common case in extrasolar planetary systems (Lee \& Peale 2003; Migaszewski \& Goździewski 2008), in artificial satellite theory (Lara et al. 2012, 2018), and in hierarchical N-body systems in general (Hamers et al. 2015; Will 2017). These types of orbits may need higher degrees in the expansion of the third-body disturbing function to provide a reasonable approximation of the dynamics even in the simplifications offered by the secular dynamics (Beaugé \& Michtchenko 2003; Libert \& Sansottera 2013; Andrade-Ines et al. 2016; Andrade-Ines \& Robutel 2018; Sansottera \& Libert 2019). This fact has motivated the recent appearance of general expressions of the expansion of the disturbing function in powers of the ratio of the disturbing and disturbed semimajor axes, including the secular terms (Laskar \& Boué 2010; Mardling 2013; Palacián et al. 2017). These expressions rely on classical elements of the Keplerian motion and apply to any eccentricity and inclination.

We constrain ourselves to the restricted approximation, in which the lower mass body does not affect the motion of the primaries. This is a reasonable approximation in artificial satellite theory as well as in some problems of Solar System dynamics, but it does not apply to the dynamics of extrasolar planetary

A brief historical review on the topic was given by Deprit (1975),
and additional details were presented by Rosengren \& Scheeres (2014). 
systems. For the restricted case, we make use of the apsidal frame formulation, which effectively displays the fast and slow components of the orbital motion, and we use perturbation theory to remove the short-period components of the third-body disturbing function. The third-body direction is assumed to be a known function of time, given by an ephemeris, but we avoid time-related issues in the averaging by constraining ourselves to the usual case in which the position of the third body can be taken as fixed during one orbital period. Instead of relying on the classical Hansen expansions or related eccentricity functions (Hansen 1855; Kaula 1962; Giacaglia 1974; Lane 1989; Celletti et al. 2017), we average the Legendre polynomial expansion of the third-body potential in closed form of the eccentricity after the usual reformulation in terms of the eccentric anomaly (Deprit 1983; Kelly 1989).

The decoupling of short-period effects is achieved after the standard Delaunay normalization (Deprit 1982), which we extend only to the first order in the Hamiltonian perturbation approach; that is, we do not consider the possible coupling between the different terms in which the third-body perturbation is expanded. However, while the normalization is properly carried out in Delaunay canonical variables, which are the actionangle variables of the Kepler problem, the canonical variables related to the Keplerian elements are collected in vectorial form throughout the whole normalization procedure. In this way, we obtain alternative, general expressions for the expansion of the third-body disturbing function in mean vectorial elements up to an arbitrary degree. For completeness, the generating function of the infinitesimal contact transformation from mean to osculating elements is also provided in vectorial elements up to an arbitrary degree of the expansion.

The Hamilton equations of the mean elements Hamiltonian are then computed to obtain analogous general expressions for the variation equations of the flow in Delaunay (mean) elements, which, as expected, are flawed by the appearance of the eccentricity and the sine of the inclination as divisors. Singularities are avoided by reformulating the flow in different sets of canonical and noncanonical variables. In particular, we provide vectorial, nonsingular expressions for the variation equations of the eccentricity and angular momentum vectors in mean elements. These expressions can be used to extend to an arbitrary degree existing lower-order truncations in the literature (Allan 1962; Katz et al. 2011).

\section{Third-body disturbing potential}

Let $(O, \boldsymbol{i}, \boldsymbol{j}, \boldsymbol{k})$ be an orthonormal frame with origin $O$ in the center of mass of a central attracting body and fixed directions defined by the unit vectors $\boldsymbol{i}, \boldsymbol{j}, \boldsymbol{k}$. Let $\boldsymbol{r}$ and $\boldsymbol{r}_{\star}$ be the position vectors of a massless and a massive body, respectively, of modulus $r=\|\boldsymbol{r}\|$ and $r_{\star}=\left\|\boldsymbol{r}_{\star}\right\|$. When $r \ll r_{\star}$, the third-body disturbing potential

$\mathcal{V}_{\star}=-\frac{\mu_{\star}}{r_{\star}}\left(\frac{r_{\star}}{\left\|\boldsymbol{r}-\boldsymbol{r}_{\star}\right\|}-\frac{\boldsymbol{r} \cdot \boldsymbol{r}_{\star}}{r_{\star}^{2}}\right)$,

where $\mu_{\star}$ is the third-body gravitational parameter, is customarily replaced by the Legendre polynomial expansion

$\mathcal{V}_{\star}=-\frac{n_{\star}^{2} a_{\star}^{3}}{r_{\star}} \chi_{\star} \sum_{i \geq 2} \frac{r^{i}}{r_{\star}^{i}} P_{i}\left(\cos \psi_{\star}\right)$,

in which $\mu_{\star}$ has been rewritten in terms of the mean motion $n_{\star}$ and the semimajor axis $a_{\star}$ of the third-body orbit relative to the central body, $\chi_{\star}$ is the mass ratio of the system, $\cos \psi_{\star}=\hat{\boldsymbol{r}} \cdot \hat{\boldsymbol{r}}_{\star}$, with $\hat{\boldsymbol{r}}=\boldsymbol{r} / r$ and $\hat{\boldsymbol{r}}_{\star}=\boldsymbol{r}_{\star} / r_{\star}$, and the Legendre polynomials $P_{i}$ are given by the usual expansion of Rodrigues' formula,

$P_{i}\left(\cos \psi_{\star}\right)=\frac{1}{2^{i}} \sum_{l=0}^{\lfloor i / 2\rfloor}(-1)^{l}\left(\begin{array}{l}i \\ l\end{array}\right)\left(\begin{array}{c}2 i-2 l \\ i\end{array}\right) \cos ^{i-2 l} \psi_{\star}$,

with $\lfloor i / 2\rfloor$ denoting the integer part of the division $i / 2$. We recall that as usual, the term $-\mu_{\star} / r_{\star}$ is neglected in Eq. (2) because it does not contribute to the disturbing acceleration of the massless body.

We define now the apsidal moving frame $(O, \hat{\boldsymbol{e}}, \boldsymbol{b}, \boldsymbol{n})$ with the same origin as before and the unit vectors $\boldsymbol{n}$ in the direction of the (instantaneous) angular momentum vector (per unit of mass),

$\boldsymbol{G}=G \boldsymbol{n}=\boldsymbol{r} \times \boldsymbol{v}$,

where $G=\|\boldsymbol{G}\|$ and $\boldsymbol{v}=\mathrm{d} \boldsymbol{r} / \mathrm{d} t$, with $t$ denoting time. The unit vector $\hat{\boldsymbol{e}}$ has the direction of the (instantaneous) eccentricity vector,

$\boldsymbol{e}=e \hat{\boldsymbol{e}}=\frac{\boldsymbol{v} \times \boldsymbol{G}}{\mu}-\frac{\boldsymbol{r}}{r}$

where $e=\|\boldsymbol{e}\|$ is the (instantaneous) eccentricity of the orbit of the massless body, and $\mu$ is the gravitational parameter of the central body. Finally, the unit vector

$\boldsymbol{b}=\boldsymbol{n} \times \hat{\boldsymbol{e}}$

defines the binormal direction, in this way completing a direct orthonormal frame.

For convenience, we define the unit vector $\boldsymbol{\ell}$ in the direction of the ascending node, given by

$\boldsymbol{k} \times \boldsymbol{n}=\boldsymbol{\ell} \sin I$,

where

$I=\arccos (\boldsymbol{k} \cdot \boldsymbol{n})$,

is the inclination angle of the (instantaneous) orbital plane with respect to the $(\boldsymbol{i}, \boldsymbol{j})$ plane. In addition,

$\boldsymbol{\ell} \cdot \hat{\boldsymbol{e}}=\cos \omega, \quad \boldsymbol{\ell} \cdot \boldsymbol{b}=-\sin \omega$,

where $\omega$ is the argument of the pericenter. Then, it is simple to check that

$\hat{\boldsymbol{e}} \cdot \boldsymbol{k}=\sin I \sin \omega=-\boldsymbol{\ell} \cdot \boldsymbol{b} \sin I$,

$\boldsymbol{b} \cdot \boldsymbol{k}=\sin I \cos \omega=\boldsymbol{\ell} \cdot \hat{\boldsymbol{e}} \sin I$.

Finally, by denoting with $h$ the longitude of the node, the components of the apsidal frame in the inertial frame are given by the rotations

$(\hat{\boldsymbol{e}}, \boldsymbol{b}, \boldsymbol{n})=R_{3}(-h) R_{1}(-I) R_{3}(-\omega)$,

where

$R_{1}(\alpha)=\left(\begin{array}{ccc}1 & 0 & 0 \\ 0 & \cos \alpha & \sin \alpha \\ 0 & -\sin \alpha & \cos \alpha\end{array}\right)$,

$R_{3}(\alpha)=\left(\begin{array}{ccc}\cos \alpha & \sin \alpha & 0 \\ -\sin \alpha & \cos \alpha & 0 \\ 0 & 0 & 1\end{array}\right)$

are standard rotation matrices. 
When the direction of the massless body is given by its components in the apsidal frame,

$\hat{\boldsymbol{r}}=\hat{\boldsymbol{e}} \cos f+\boldsymbol{b} \sin f$,

with $f$ being the true anomaly, we obtain

$\cos \psi_{\star}=\left(\hat{\boldsymbol{e}} \cdot \hat{\boldsymbol{r}}_{\star}\right) \cos f+\left(\boldsymbol{b} \cdot \hat{\boldsymbol{r}}_{\star}\right) \sin f$,

which immediately discloses the short-period terms affecting Eq. (3).

However, the short-period terms of the third-body disturbing potential in Eq. (2) are not limited to those contributed by the Legendre polynomials, and they are better handled when written in terms of the eccentric anomaly $u$, in contrast to the true anomaly. This is done using the geometric relations

$\sin f=\frac{a}{r} \eta \sin u, \quad \cos f=\frac{a}{r}(\cos u-e)$,

where the eccentricity function $\eta=\left(1-e^{2}\right)^{1 / 2}$ is used for convenience. We recall also that

$r=a(1-e \cos u)$,

and after some rearrangement, we obtain

$\mathcal{V}_{\star}=-\frac{\mu}{2 a} \chi_{\star} \frac{n_{\star}^{2}}{n^{2}} \frac{a_{\star}^{3}}{r_{\star}^{3}} \frac{a}{r} \sum_{i \geq 2} \frac{1}{2^{i-1}} \frac{a^{i-2}}{r_{\star}^{i-2}} V_{i}$,

in which the nondimensional functions $V_{i}$ take the form

$$
\begin{aligned}
V_{i}= & \sum_{l=0}^{\lfloor i / 2\rfloor}(-1)^{l}\left(\begin{array}{l}
i \\
l
\end{array}\right)\left(\begin{array}{c}
2 i-2 l \\
i
\end{array}\right) \frac{r^{2 l+1}}{a^{2 l+1}}\left[\left(\hat{\boldsymbol{e}} \cdot \hat{\boldsymbol{r}}_{\star}\right)(\cos u-e)\right. \\
& \left.+\left(\boldsymbol{b} \cdot \hat{\boldsymbol{r}}_{\star}\right) \eta \sin u\right]^{i-2 l}
\end{aligned}
$$

The reasons for the particular arrangement of Eq. (17), in which we excluded the coefficient $a / r$ from the summation, is that the removal of short-period terms from $\mathcal{V}_{\star}$, which is carried out by Eq. (24), is easily achieved in closed form of the eccentricity by taking advantage of the differential relation between the mean and true anomalies.

Next, we substitute Eq. (16) into Eq. (18) and use the binomial expansion to obtain

$\frac{r^{2 l+1}}{a^{2 l+1}}=(1-e \cos u)^{2 l+1}=\sum_{j=0}^{2 l+1}\left(\begin{array}{c}2 l+1 \\ j\end{array}\right)(-1)^{j} e^{j} \cos ^{j} u$.

Analogously, the binomial expansion is applied to the last term in the right side of Eq. (18), to yield

$$
\begin{aligned}
& {\left[\left(\hat{\boldsymbol{e}} \cdot \hat{\boldsymbol{r}}_{\star}\right)(\cos u-e)+\left(\boldsymbol{b} \cdot \hat{\boldsymbol{r}}_{\star}\right) \eta \sin u\right]^{i-2 l}} \\
& \quad=\sum_{k=0}^{i-2 l}\left(\begin{array}{c}
i-2 l \\
k
\end{array}\right)\left(\hat{\boldsymbol{e}} \cdot \hat{\boldsymbol{r}}_{\star}\right)^{k}(\cos u-e)^{k}\left(\boldsymbol{b} \cdot \hat{\boldsymbol{r}}_{\star}\right)^{i-2 l-k} \eta^{i-2 l-k} \sin ^{i-2 l-k} u,
\end{aligned}
$$

where

$(\cos u-e)^{k}=\sum_{m=0}^{k}\left(\begin{array}{l}k \\ m\end{array}\right)(-1)^{m} e^{m} \cos ^{k-m} u$.

Plugging Eqs. (19)-(21) into Eq. (18), we finally obtain

$$
\begin{aligned}
V_{i}= & \sum_{l=0}^{\lfloor i / 2\rfloor}\left(\begin{array}{c}
i \\
l
\end{array}\right)\left(\begin{array}{c}
2 i-2 l \\
i
\end{array}\right) \sum_{j=0}^{2 l+1}\left(\begin{array}{c}
2 l+1 \\
j
\end{array}\right) \sum_{k=0}^{i-2 l}\left(\begin{array}{c}
i-2 l \\
k
\end{array}\right) \sum_{m=0}^{k}\left(\begin{array}{l}
k \\
m
\end{array}\right) e^{j+m} \\
& \times(-1)^{j+l+m} \eta^{i-2 l-k}\left(\hat{\boldsymbol{e}} \cdot \hat{\boldsymbol{r}}_{\star}\right)^{k}\left(\boldsymbol{b} \cdot \hat{\boldsymbol{r}}_{\star}\right)^{i-2 l-k} \\
& \times \cos ^{j+k-m} u \sin ^{i-2 l-k} u .
\end{aligned}
$$

\section{Short-period averaging}

Because third-body perturbations derive from the potential in Eq. (2), we can take advantage of the Hamiltonian formalism. Thus

$\mathcal{H}=-\frac{\mu}{2 a}+\mathcal{V}_{\star}$,

where $\mathcal{H}$ must be expressed in some set of canonical variables. In particular, we use Delaunay variables $(\ell, g, h, L, G, H)$, where the coordinates are the mean anomaly $\ell$, the argument of the periapsis $g$, and the longitude of the node $h$, and their conjugate momenta are the Delaunay action $L=\sqrt{\mu a}$, the modulus of the angular momentum vector $G$, and its projection along the $\boldsymbol{k}$ direction $H$, respectively. A modern derivation of this useful set of variables can be found, for instance, in Lara (2016).

Disregarding short-period effects, the orbit evolution is customarily studied in mean elements $\left(\ell^{\prime}, g^{\prime}, h^{\prime}, L^{\prime}, G^{\prime}, H^{\prime}\right)$, which aim to represent the average value of the true osculating variables. The transformation from mean to osculating variables is found using the tools of perturbation theory. To avoid timedependency issues introduced by the third-body ephemeris $\hat{\boldsymbol{r}}_{\star} \equiv \hat{\boldsymbol{r}}_{\star}(t)$, we used the extended phase-space formulation. In particular, we relied on the Lie transforms method (Hori 1966; Deprit 1969), which is considered standard and is thoroughly described in the literature (see, e.g., Meyer \& Hall 1992; Boccaletti \& Pucacco 2002). The details of the transformation will be presented elsewhere, and we focus here on the averaging of the disturbing potential. However, because its computation is immediate once the disturbing potential has been averaged, we also provide the generating function from which the short-period corrections are derived.

The short-period elimination is carried out in closed form of the eccentricity. It is effectively achieved with the help of the differential relation between the eccentric and true anomalies $\mathrm{d} \ell=(1-e \cos u) \mathrm{d} u$, which is obtained by differentiating Kepler's equation. Thus, because of Eq. (16),

$\left\langle\mathcal{V}_{\star}\right\rangle_{\ell}=\frac{1}{2 \pi} \int_{0}^{2 \pi} \mathcal{V}_{\star} \mathrm{d} \ell=\frac{1}{2 \pi} \int_{0}^{2 \pi} \mathcal{V}_{\star} \frac{r}{a} \mathrm{~d} u$,

from which, after replacing Eq. (17), we obtain

$\left\langle\mathcal{V}_{\star}\right\rangle_{\ell}=-\chi_{\star} \frac{\mu}{2 a} \frac{n_{\star}^{2}}{n^{2}} \frac{a_{\star}^{3}}{r_{\star}^{3}} \sum_{i \geq 2} \frac{1}{2^{i-1}} \frac{a^{i-2}}{r_{\star}^{i-2}}\left\langle V_{i}\right\rangle_{u}$,

where

$\left\langle V_{i}\right\rangle_{u}=\frac{1}{2 \pi} \int_{0}^{2 \pi} V_{i} \mathrm{~d} u$.

In view of Eq. (22), the only terms that remain below the integral symbol of Eq. (26) are of the form $\sin ^{a} u \cos ^{b} u$ with $a$ and $b$ integers. Terms of this type are easily integrated when they are expressed as Fourier series in $u$. Straightforward computations using standard relations between exponentials and circular functions (see, e.g., Kaula 1966), yield

$$
\begin{aligned}
\cos ^{j+k-m} u \sin ^{i-2 l-k} u= & \frac{(-\mathbf{i})^{i-k-2 l}}{2^{i+j-m-2 l}} \sum_{q=0}^{i-k-2 l} \sum_{t=0}^{j+k-m}\left(\begin{array}{c}
i-k-2 l \\
q
\end{array}\right)(-1)^{q} \\
& \times\left(\begin{array}{c}
j+k-m \\
t
\end{array}\right) \\
& \times[\cos 2(\tilde{q}-q) u+\mathbf{i} \sin 2(\tilde{q}-q) u],
\end{aligned}
$$


where $\mathbf{i}$ notes the imaginary unit, and we abbreviated

$\tilde{q}=\frac{i+j-m}{2}-l-t$

Now, it becomes obvious that the only nonperiodic terms of Eq. (27)

$\Xi_{j+k-m}^{i-2 l-k}=\left\langle\cos ^{j+k-m} u \sin ^{i-2 l-k} u\right\rangle_{u}$,

are those such that $q=\tilde{q}$, a condition that is only accomplished when $i+j-m$ is even, which in turn implies that $i+j+m$ is also even. In addition, because Eq. (27) must be free from imaginary terms, $i-k$, the exponent of the imaginary unit, must be even in the case of nonperiodic terms, yielding the numeric coefficient

$\Xi_{2 a}^{2 b}=\frac{1}{2^{2 a+2 b}} \sum_{t=0}^{2 a}\left(\begin{array}{c}2 b \\ b+a-t\end{array}\right)(-1)^{a-t}\left(\begin{array}{c}2 a \\ t\end{array}\right)$,

where $a=\frac{1}{2}(j+k-m)$ and $b=\frac{1}{2}(i-k)-l$ are integer numbers.

On the other hand, $i-k$ even allows us to replace $\eta^{i-k-2 l}$ by the binomial expansion of $\left(1-e^{2}\right)^{\frac{1}{2}(i-k-2 l)}$. Thus, replacing

$e^{m+j}\left(1-e^{2}\right)^{\frac{i-k}{2}-l}=\sum_{q=0}^{\frac{i-k}{2}-l}\left(\begin{array}{c}\frac{i-k}{2}-l \\ q\end{array}\right)(-1)^{q} e^{m+j+2 q}$,

into, Eq. (26), we finally obtain

$$
\begin{aligned}
\left\langle V_{i}\right\rangle_{u}= & \sum_{l=0}^{\lfloor i / 2\rfloor}\left(\begin{array}{c}
i \\
l
\end{array}\right)\left(\begin{array}{c}
2 i-2 l \\
i
\end{array}\right) \sum_{k=0}^{i-2 l}\left(\begin{array}{c}
i-2 l \\
k
\end{array}\right) \sum_{j=0}^{2 l+1}\left(\begin{array}{c}
2 l+1 \\
j
\end{array}\right) \sum_{m=0}^{k}\left(\begin{array}{c}
k \\
m
\end{array}\right) \Xi_{j+k-m}^{i-2 l-k} \\
& \times \sum_{q=0}^{\frac{i-k}{2}-l}\left(\begin{array}{c}
\frac{i-k}{2}-l \\
q
\end{array}\right)(-1)^{i-l+q} e^{m+j+2 q}\left(\hat{\boldsymbol{e}} \cdot \hat{\boldsymbol{r}}_{\star}\right)^{k}\left(\boldsymbol{b} \cdot \hat{\boldsymbol{r}}_{\star}\right)^{i-2 l-k},
\end{aligned}
$$

thus completing the calculation of Eq. (25).

The generating function of the infinitesimal contact transformation leading to the averaging is computed from the usual relation

$\mathcal{W}=\frac{1}{n} \int\left(\mathcal{V}_{\star}-\left\langle\mathcal{V}_{\star}\right\rangle_{\ell}\right) \mathrm{d} \ell$

That is,

$$
\begin{aligned}
\mathcal{W} & =-\chi_{\star} L \frac{n_{\star}^{2}}{n^{2}} \frac{a_{\star}^{3}}{r_{\star}^{3}} \sum_{i \geq 2} \frac{1}{2^{i}} \frac{a^{i-2}}{r_{\star}^{i-2}} \int\left(\frac{a}{r} V_{i}-\left\langle V_{i}\right\rangle_{u}\right) \mathrm{d} \ell \\
& =-\chi_{\star} L \frac{n_{\star}^{2}}{n^{2}} \frac{a_{\star}^{3}}{r_{\star}^{3}} \sum_{i \geq 2} \frac{1}{2^{i}} \frac{a^{i-2}}{r_{\star}^{i-2}}\left(-\left\langle V_{i}\right\rangle_{u} \ell+\int V_{i} \mathrm{~d} u\right),
\end{aligned}
$$

which can be written in the form

$\mathcal{W}=-\chi_{\star} L \frac{n_{\star}^{2}}{n^{2}} \frac{a_{\star}^{3}}{r_{\star}^{3}} \sum_{i \geq 2} \frac{1}{2^{i}} \frac{a^{i-2}}{r_{\star}^{i-2}}\left[\left\langle V_{i}\right\rangle_{u}(u-\ell)+\int\left(V_{i}-\left\langle V_{i}\right\rangle_{u}\right) \mathrm{d} u\right]$,

where $u-\ell=e \sin u$, from the Kepler equation, and the integrand of the last term in the square brackets is only composed of periodic terms in the eccentric anomaly. Then, after replacing Eq. (27) into Eq. (22), we solve the indefinite integral by keeping all the trigonometric terms except for those that make $i+j-m-2 l-2 q-2 t=0$. Namely,

$$
\begin{aligned}
\int\left(V_{i}-\left\langle V_{i}\right\rangle_{u}\right) \mathrm{d} u= & \sum_{l=0}^{\lfloor i / 2\rfloor}\left(\begin{array}{c}
i \\
l
\end{array}\right)\left(\begin{array}{c}
2 i-2 l \\
i
\end{array}\right) \sum_{j=0}^{2 l+1}\left(\begin{array}{c}
2 l+1 \\
j
\end{array}\right) \sum_{k=0}^{i-2 l}\left(\begin{array}{c}
i-2 l \\
k
\end{array}\right) \\
& \times \sum_{m=0}^{k}\left(\begin{array}{l}
k \\
m
\end{array}\right) e^{m+j} \eta^{i-2 l-k}\left(\hat{\boldsymbol{e}} \cdot \hat{\boldsymbol{r}}_{\star}\right)^{k}\left(\boldsymbol{b} \cdot \hat{\boldsymbol{r}}_{\star}\right)^{i-2 l-k} \\
& \times(-1)^{j+l+m} \frac{(-\mathbf{i})^{i-2 l-k}}{2^{i-2 l+j-m}} \sum_{q=0}^{i-2 l-k} \sum_{t=0}^{j+k-m}\left(\begin{array}{c}
i-2 l-k \\
q
\end{array}\right) \\
& \times\left(\begin{array}{c}
j+k-m \\
t
\end{array}\right)(-1)^{q} \\
& \times \frac{\sin 2(\tilde{q}-q) u-\mathbf{i} \cos 2(\tilde{q}-q) u}{2(\tilde{q}-q)}
\end{aligned}
$$

where $\sin 2(\tilde{q}-q) u-\mathbf{i} \cos 2(\tilde{q}-q) u=-\mathbf{i}(\cos u+\mathbf{i} \sin u)^{2(\tilde{q}-q)}$ from which, using Eq. (15),

$\sin 2(\tilde{q}-q) u-\mathbf{i} \cos 2(\tilde{q}-q) u=-\mathbf{i}\left(e+\frac{\boldsymbol{r} \cdot \hat{\boldsymbol{e}}}{a}+\mathbf{i} \frac{\boldsymbol{r} \cdot \boldsymbol{b}}{a \eta}\right)^{2(\tilde{q}-q)}$.

Finally, the Hamiltonian in mean elements, up to higher order effects, is obtained by replacing osculating by mean elements into both the Keplerian $-\mu /(2 a)$ and the third-body averaged potential, Eq. (25), to yield

$\mathcal{K}=-\frac{\mu}{2 a}+\left\langle\mathcal{V}_{\star}\right\rangle_{\ell}$,

where now all the symbols appearing in $\mathcal{K}$ are functions of the Delaunay prime elements.

\section{Hamilton equations of the averaged flow}

The flow in mean (prime) elements is obtained from the Hamilton equations. Because the mean anomaly has been removed by the perturbation approach up to the truncation order of the perturbation solution, the prime Delaunay action is a constant that decouples the reduced flow

$$
\begin{aligned}
& \frac{\mathrm{d} g^{\prime}}{\mathrm{d} t}=\frac{\partial \mathcal{K}}{\partial G^{\prime}}=\frac{\partial\left\langle\mathcal{V}_{\star}\right\rangle_{\ell}}{\partial G^{\prime}}, \\
& \frac{\mathrm{d} G^{\prime}}{\mathrm{d} t}=-\frac{\partial \mathcal{K}}{\partial g^{\prime}}=-\frac{\partial\left\langle\mathcal{V}_{\star}\right\rangle_{\ell}}{\partial g^{\prime}}, \\
& \frac{\mathrm{d} h^{\prime}}{\mathrm{d} t}=\frac{\partial \mathcal{K}}{\partial H^{\prime}}=\frac{\partial\left\langle\mathcal{V}_{\star}\right\rangle_{\ell}}{\partial H^{\prime}}, \\
& \frac{\mathrm{d} H^{\prime}}{\mathrm{d} t}=-\frac{\partial \mathcal{K}}{\partial h^{\prime}}=-\frac{\partial\left\langle\mathcal{V}_{\star}\right\rangle_{\ell}}{\partial h^{\prime}},
\end{aligned}
$$

from the integration of the variation of the prime mean anomaly,

$$
\frac{\mathrm{d} \ell^{\prime}}{\mathrm{d} t}=\frac{\partial \mathcal{K}}{\partial L^{\prime}}=n+\frac{\partial\left\langle\mathcal{V}_{\star}\right\rangle_{\ell}}{\partial L^{\prime}},
$$

which is computed by indefinite integration after solving the reduced system of Eqs. (39)-(42).

In fact, in view of Eqs. (25) and (32), computing the right sides of the variation Eqs. (39)-(42) only requires the corresponding partial derivatives of the function

$\alpha_{\sigma, k, p}=e^{p}\left(\hat{\boldsymbol{e}} \cdot \hat{\boldsymbol{r}}_{\star}\right)^{k}\left(\boldsymbol{b} \cdot \hat{\boldsymbol{r}}_{\star}\right)^{\sigma-k}, \quad \sigma=i-2 l, \quad p=m+j+2 q$. 
In Eq. (44), both direction vectors $\hat{\boldsymbol{e}}$ and $\boldsymbol{b}$ depend on the orbital inclination $I$, the argument of the periapsis $g$, and the longitude of the node $h$, as shown in Eq. (12).

The required partial derivatives of $\alpha_{\sigma, k, p}$ with respect to the Delaunay variables are computed using the chain rule:

$$
\begin{aligned}
\frac{\partial \alpha_{\sigma, k, p}}{\partial G}= & \frac{\partial \alpha_{\sigma, k, p}}{\partial e} \frac{\partial e}{\partial G}+\frac{\partial \alpha_{\sigma, k, p}}{\partial\left(\hat{\boldsymbol{e}} \cdot \hat{\boldsymbol{r}}_{\star}\right)}\left(\frac{\partial \hat{\boldsymbol{e}}}{\partial I} \frac{\partial I}{\partial G}\right) \cdot \hat{\boldsymbol{r}}_{\star} \\
& +\frac{\partial \alpha_{\sigma, k, p}}{\partial\left(\boldsymbol{b} \cdot \hat{\boldsymbol{r}}_{\star}\right)}\left(\frac{\partial \boldsymbol{b}}{\partial I} \frac{\partial I}{\partial G}\right) \cdot \hat{\boldsymbol{r}}_{\star}, \\
\frac{\partial \alpha_{\sigma, k, p}}{\partial H}= & \frac{\partial \alpha_{\sigma, k, p}}{\partial\left(\hat{\boldsymbol{e}} \cdot \hat{\boldsymbol{r}}_{\star}\right)}\left(\frac{\partial \hat{\boldsymbol{e}}}{\partial I} \frac{\partial I}{\partial H}\right) \cdot \hat{\boldsymbol{r}}_{\star} \\
& +\frac{\partial \alpha_{\sigma, k, p}}{\partial\left(\boldsymbol{b} \cdot \hat{\boldsymbol{r}}_{\star}\right)}\left(\frac{\partial \boldsymbol{b}}{\partial I} \frac{\partial I}{\partial H}\right) \cdot \hat{\boldsymbol{r}}_{\star}, \\
\frac{\partial \alpha_{\sigma, k, p}}{\partial g}= & \frac{\partial \alpha_{\sigma, k, p}}{\partial\left(\hat{\boldsymbol{e}} \cdot \hat{\boldsymbol{r}}_{\star}\right)} \frac{\partial \hat{\boldsymbol{e}}}{\partial g} \cdot \hat{\boldsymbol{r}}_{\star}+\frac{\partial \alpha_{\sigma, k, p}}{\partial\left(\boldsymbol{b} \cdot \hat{\boldsymbol{r}}_{\star}\right)} \frac{\partial \boldsymbol{b}}{\partial g} \cdot \hat{\boldsymbol{r}}_{\star}, \\
\frac{\partial \alpha_{\sigma, k, p}}{\partial h}= & \frac{\partial \alpha_{\sigma, k, p}}{\partial\left(\hat{\boldsymbol{e}} \cdot \hat{\boldsymbol{r}}_{\star}\right)} \frac{\partial \hat{\boldsymbol{e}}}{\partial h} \cdot \hat{\boldsymbol{r}}_{\star}+\frac{\partial \alpha_{\sigma, k, p}}{\partial\left(\boldsymbol{b} \cdot \hat{\boldsymbol{r}}_{\star}\right)} \frac{\partial \boldsymbol{b}}{\partial h} \cdot \hat{\boldsymbol{r}}_{\star} .
\end{aligned}
$$

Because $c \equiv \cos I=H / G, s \equiv \sin I=\left(1-c^{2}\right)^{1 / 2}$, and $e=$ $\left(1-G^{2} / L^{2}\right)^{1 / 2}$, we easily obtain

$\frac{\partial e}{\partial G}=-\frac{1}{G} \frac{\eta^{2}}{e}, \quad \frac{\partial I}{\partial H}=-\frac{1}{G} \frac{1}{s}, \quad \frac{\partial I}{\partial G}=\frac{1}{G} \frac{c}{s}$.

In addition, because the effect of a differential rotation about the axis $\boldsymbol{n}$ (resp. $\boldsymbol{\ell}, \boldsymbol{k}$ ) is an infinitesimal increase of the angle $g$ (resp. $I, h)$, we find

$$
\frac{\partial \hat{\boldsymbol{e}}}{\partial g}=\boldsymbol{n} \times \hat{\boldsymbol{e}}=\boldsymbol{b}, \quad \frac{\partial \hat{\boldsymbol{e}}}{\partial h}=\boldsymbol{k} \times \hat{\boldsymbol{e}}, \quad \frac{\partial \hat{\boldsymbol{e}}}{\partial I}=\boldsymbol{\ell} \times \hat{\boldsymbol{e}}=\frac{1}{S}(\hat{\boldsymbol{e}} \cdot \boldsymbol{k}) \boldsymbol{n},
$$

and

$$
\frac{\partial \boldsymbol{b}}{\partial g}=\boldsymbol{n} \times \boldsymbol{b}=-\hat{\boldsymbol{e}}, \quad \frac{\partial \boldsymbol{b}}{\partial h}=\boldsymbol{k} \times \boldsymbol{b}, \quad \frac{\partial \boldsymbol{b}}{\partial I}=\boldsymbol{\ell} \times \boldsymbol{b}=\frac{1}{s}(\boldsymbol{b} \cdot \boldsymbol{k}) \boldsymbol{n} .
$$

Thus, straightforward computations yield

$$
\begin{aligned}
\frac{\partial \alpha_{\sigma, k, p}}{\partial G}= & -\frac{1}{G} \frac{\eta^{2}}{e}\left[p e^{p-1}\right]\left(\hat{\boldsymbol{e}} \cdot \hat{\boldsymbol{r}}_{\star}\right)^{k}\left(\boldsymbol{b} \cdot \hat{\boldsymbol{r}}_{\star}\right)^{\sigma-k} \\
& +\frac{1}{G} \frac{c}{S} e^{p}\left[k\left(\hat{\boldsymbol{e}} \cdot \hat{\boldsymbol{r}}_{\star}\right)^{k-1}\right]\left(\boldsymbol{b} \cdot \hat{\boldsymbol{r}}_{\star}\right)^{\sigma-k}\left(\boldsymbol{n} \cdot \hat{\boldsymbol{r}}_{\star}\right) \frac{\hat{\boldsymbol{e}} \cdot \boldsymbol{k}}{S} \\
& +\frac{1}{G} \frac{c}{S} e^{p}\left(\hat{\boldsymbol{e}} \cdot \hat{\boldsymbol{r}}_{\star}\right)^{k}\left[(\sigma-k)\left(\boldsymbol{b} \cdot \hat{\boldsymbol{r}}_{\star}\right)^{\sigma-k-1}\right]\left(\boldsymbol{n} \cdot \hat{\boldsymbol{r}}_{\star}\right) \frac{\boldsymbol{b} \cdot \boldsymbol{k}}{s},
\end{aligned}
$$

$$
\begin{aligned}
\frac{\partial \alpha_{\sigma, k, p}}{\partial H}= & -\frac{1}{G} \frac{1}{S} e^{p}\left[k\left(\hat{\boldsymbol{e}} \cdot \hat{\boldsymbol{r}}_{\star}\right)^{k-1}\right]\left(\boldsymbol{b} \cdot \hat{\boldsymbol{r}}_{\star}\right)^{\sigma-k}\left(\boldsymbol{n} \cdot \hat{\boldsymbol{r}}_{\star}\right) \frac{\hat{\boldsymbol{e}} \cdot \boldsymbol{k}}{s} \\
& -\frac{1}{G} \frac{1}{S} e^{p}\left(\hat{\boldsymbol{e}} \cdot \hat{\boldsymbol{r}}_{\star}\right)^{k}\left[(\sigma-k)\left(\boldsymbol{b} \cdot \hat{\boldsymbol{r}}_{\star}\right)^{\sigma-k-1}\right]\left(\boldsymbol{n} \cdot \hat{\boldsymbol{r}}_{\star}\right) \frac{\boldsymbol{b} \cdot \boldsymbol{k}}{s},
\end{aligned}
$$

$$
\begin{aligned}
\frac{\partial \alpha_{\sigma, k, p}}{\partial g}= & e^{p}\left\{\left[k\left(\hat{\boldsymbol{e}} \cdot \hat{\boldsymbol{r}}_{\star}\right)^{k-1}\right]\left(\boldsymbol{b} \cdot \hat{\boldsymbol{r}}_{\star}\right)^{\sigma-k+1}\right. \\
& \left.-\left(\hat{\boldsymbol{e}} \cdot \hat{\boldsymbol{r}}_{\star}\right)^{k+1}\left[(\sigma-k)\left(\boldsymbol{b} \cdot \hat{\boldsymbol{r}}_{\star}\right)^{\sigma-k-1}\right]\right\}, \\
\frac{\partial \alpha_{\sigma, k, p}}{\partial h}= & e^{p}\left\{\left[k\left(\hat{\boldsymbol{e}} \cdot \hat{\boldsymbol{r}}_{\star}\right)^{k-1}\right]\left(\boldsymbol{b} \cdot \hat{\boldsymbol{r}}_{\star}\right)^{\sigma-k}\left(\hat{\boldsymbol{e}} \times \hat{\boldsymbol{r}}_{\star}\right)\right. \\
& \left.+\left(\hat{\boldsymbol{e}} \cdot \hat{\boldsymbol{r}}_{\star}\right)^{k}\left[(\sigma-k)\left(\boldsymbol{b} \cdot \hat{\boldsymbol{r}}_{\star}\right)^{\sigma-k-1}\right]\left(\boldsymbol{b} \times \hat{\boldsymbol{r}}_{\star}\right)\right\} \cdot \boldsymbol{k},
\end{aligned}
$$

where the dispensable square brackets in these equations are used to emphasize that corresponding enclosed terms never introduce denominators.

\section{Long-term flow in mean vectorial elements}

As expected from the singularities of Delaunay variables, Eqs. (52) and (53) are singular in the case of circular and equatorial orbits. However, these singularities are of virtual nature (Henrard 1974) and may be removed when the equations of the flow are represented in other sets of canonical or noncanonical variables. In particular, the variations experienced by the angular momentum vector and the eccentricity vector under third-body perturbations are free from singularities and provide a general, compact, and elegant way of presenting the variation of the mean elements.

When the mean angular momentum vector is scaled by $L^{\prime}$, the variations of the mean vectorial elements $\boldsymbol{h}=$ $\boldsymbol{G} / L^{\prime}=\eta \boldsymbol{n}$ and $\boldsymbol{e}=e \hat{\boldsymbol{e}}$ take the neat symmetric form (Milankovitch 1941; Allan \& Ward 1963; Allan \& Cook 1964; Rosengren \& Scheeres 2014)

$\begin{aligned} \frac{\mathrm{d} \boldsymbol{h}}{\mathrm{d} t} & =\boldsymbol{h} \times \frac{\partial \mathcal{R}}{\partial \boldsymbol{h}}+\boldsymbol{e} \times \frac{\partial \mathcal{R}}{\partial \boldsymbol{e}}, \\ \frac{\mathrm{d} \boldsymbol{e}}{\mathrm{d} t} & =\boldsymbol{e} \times \frac{\partial \mathcal{R}}{\partial \boldsymbol{h}}+\boldsymbol{h} \times \frac{\partial \mathcal{R}}{\partial \boldsymbol{e}},\end{aligned}$

in which

$\mathcal{R}=-\frac{1}{L^{\prime}}\left\langle\mathcal{V}_{\star}\right\rangle_{\ell}$,

and $\left\langle\mathcal{V}_{\star}\right\rangle_{\ell}$ is given in Eq. (25). Furthermore, derivation of Eqs. (56)-(57) is trivial after minor rearrangement of Eq. (32) to replace the directions $\hat{\boldsymbol{e}}$ and $\boldsymbol{n}$ by the nondimensional magnitudes $\boldsymbol{e}$ and $\boldsymbol{h}$, respectively.

Thus, instead of replacing Eq. (31) in Eq. (32), the latter is written in the more convenient form

$$
\begin{aligned}
\left\langle V_{i}\right\rangle_{u}= & \sum_{l=0}^{\lfloor i / 2\rfloor}\left(\begin{array}{c}
i \\
l
\end{array}\right)\left(\begin{array}{c}
2 i-2 l \\
i
\end{array}\right) \sum_{j=0}^{2 l+1}\left(\begin{array}{c}
2 l+1 \\
j
\end{array}\right) \sum_{k=0}^{i-2 l}\left(\begin{array}{c}
i-2 l \\
k
\end{array}\right) \sum_{m=0}^{k}\left(\begin{array}{l}
k \\
m
\end{array}\right) . \\
& \times \Xi_{j+k-m}^{i-2 l-k}(-1)^{j+l+m} e^{j+m-k}\left(e \hat{\boldsymbol{e}} \cdot \hat{\boldsymbol{r}}_{\star}\right)^{k}\left[\eta^{2}\left(\boldsymbol{b} \cdot \hat{\boldsymbol{r}}_{\star}\right)^{2}\right]^{\frac{i-k}{2}-l} .
\end{aligned}
$$

When now we make use of the identity

$\left(\hat{\boldsymbol{e}} \cdot \hat{\boldsymbol{r}}_{\star}\right)^{2}+\left(\boldsymbol{b} \cdot \hat{\boldsymbol{r}}_{\star}\right)^{2}+\left(\boldsymbol{n} \cdot \hat{\boldsymbol{r}}_{\star}\right)^{2}=1$,

which gives the square of the modulus of the third-body direction $\left\|\hat{\boldsymbol{r}}_{\star}\right\|=1$ when it is computed in the apsidal frame, the dependence of the averaged potential on the unit vector $\boldsymbol{b}$ in the binormal direction is replaced by a corresponding dependence on the normal direction $\boldsymbol{n}$. That is

$$
\begin{aligned}
\left\langle V_{i}\right\rangle_{u}= & \sum_{l=0}^{\lfloor i / 2\rfloor}\left(\begin{array}{c}
i \\
l
\end{array}\right)\left(\begin{array}{c}
2 i-2 l \\
i
\end{array}\right) \sum_{j=0}^{2 l+1}\left(\begin{array}{c}
2 l+1 \\
j
\end{array}\right) \sum_{k=0}^{i-2 l}\left(\begin{array}{c}
i-2 l \\
k
\end{array}\right) \sum_{m=0}^{k}\left(\begin{array}{l}
k \\
m
\end{array}\right) \\
& \times \Xi_{j+k-m}^{i-2 l-k}(-1)^{j+l+m}(\boldsymbol{e} \cdot \boldsymbol{e})^{\frac{j+m-k}{2}}\left(\boldsymbol{e} \cdot \hat{\boldsymbol{r}}_{\star}\right)^{k}\left[X-\left(\hat{\boldsymbol{e}} \cdot \hat{\boldsymbol{r}}_{\star}\right)^{2}\right]^{\frac{i-k}{2}-l},
\end{aligned}
$$

with the scalar function

$X=X\left(\boldsymbol{e}, \boldsymbol{h}, \hat{\boldsymbol{r}}_{\star}\right) \equiv 1-e^{2}+\xi^{2}-\zeta^{2}$,

where we introduced the abbreviations $\xi=\boldsymbol{e} \cdot \hat{\boldsymbol{r}}_{\star}, \zeta=\boldsymbol{h} \cdot \hat{\boldsymbol{r}}_{\star}$.

Now, applying the binomial expansion again, we find

$$
\begin{aligned}
\left\langle V_{i}\right\rangle_{u}= & \sum_{l=0}^{i i / 2\rfloor}\left(\begin{array}{c}
i \\
l
\end{array}\right)\left(\begin{array}{c}
2 i-2 l \\
i
\end{array}\right) \sum_{j=0}^{2 l+1}\left(\begin{array}{c}
2 l+1 \\
j
\end{array}\right) \sum_{k=0}^{i-2 l}\left(\begin{array}{c}
i-2 l \\
k
\end{array}\right) \sum_{m=0}^{k}\left(\begin{array}{c}
k \\
m
\end{array}\right) \sum_{s=0}^{\frac{i-k}{2}-l}\left(\begin{array}{c}
\frac{i-k}{2}-l \\
s
\end{array}\right) \\
& \times \Xi_{j+k-m}^{i-2 l-k}(-1)^{j+l+m+s}(\boldsymbol{e} \cdot \boldsymbol{e})^{\frac{j+m-k}{2}-s}\left(\boldsymbol{e} \cdot \hat{\boldsymbol{r}}_{\star}\right)^{2 s+k} X^{\frac{i-k}{2}-l-s},
\end{aligned}
$$


which only depends on $\boldsymbol{e}$ and $\boldsymbol{h}$, as desired, as well as on the direction $\hat{\boldsymbol{r}}_{\star}$ of the disturbing body.

Explicit expressions for the first terms of the Legendre polynomial expansion yield the compact expressions

$$
\begin{aligned}
& \left\langle V_{2}\right\rangle_{u}=1-3\left(2 e^{2}-5 \xi^{2}+\zeta^{2}\right), \\
& \left\langle V_{3}\right\rangle_{u}=-\frac{5}{2} \xi\left[3\left(1-8^{2}\right)-15 \zeta^{2}+35 \xi^{2}\right], \\
& \left\langle V_{4}\right\rangle_{u}=\frac{3}{4}\left\{3-20 e^{2}+80 e^{4}\right. \\
& +10\left[7\left(1-10 e^{2}\right) \xi^{2}-\left(3-10 e^{2}\right) \zeta^{2}\right] \\
& \left.+35\left(21 \xi^{4}-14 \zeta^{2} \xi^{2}+\zeta^{4}\right)\right\} \\
& \left\langle V_{5}\right\rangle_{u}=-\frac{3}{4} \xi\left\{35\left(1-8 e^{2}+40 e^{4}\right)\right. \\
& +490\left[\left(1-12 e^{2}\right) \xi^{2}-\left(1-4 e^{2}\right) \zeta^{2}\right] \\
& \left.+147\left(33 \xi^{4}-30 \zeta^{2} \xi^{2}+5 \zeta^{4}\right)\right\}, \\
& \left\langle V_{6}\right\rangle_{u}=\frac{1}{4}\left\{5\left(5-42 e^{2}+168 e^{4}-560 e^{6}\right)\right. \\
& +105\left[3\left(3-28 e^{2}+168 e^{4}\right) \xi^{2}\right. \\
& \left.-\left(5-28 e^{2}+56 e^{4}\right) \zeta^{2}\right] \\
& +315\left[\left(5-14 e^{2}\right) \zeta^{4}-18\left(3-14 e^{2}\right) \zeta^{2} \xi^{2}\right. \\
& \left.+33\left(1-14 e^{2}\right) \xi^{4}\right] \\
& \left.+231\left(429 \xi^{6}-495 \zeta^{2} \xi^{4}+135 \zeta^{4} \xi^{2}-5 \zeta^{6}\right)\right\}, \\
& \left\langle V_{7}\right\rangle_{u}=-\frac{9}{16} \xi\left\{35\left(5-48 e^{2}+224 e^{4}-896 e^{6}\right)\right. \\
& +105\left[11\left(3-32 e^{2}+224 e^{4}\right) \xi^{2}\right. \\
& \left.-3\left(15-96 e^{2}+224 e^{4}\right) \zeta^{2}\right] \\
& +231\left[143\left(1-16 e^{2}\right) \xi^{4}-110\left(3-16 e^{2}\right) \zeta^{2} \xi^{2}\right. \\
& \left.+15\left(5-16 e^{2}\right) \zeta^{4}\right] \\
& \left.+429\left(715 \xi^{6}-1001 \zeta^{2} \xi^{4}+385 \zeta^{4} \xi^{2}-35 \zeta^{6}\right)\right\}, \\
& \left\langle V_{8}\right\rangle_{u}=\frac{5}{64}\left\{7\left(35-360 e^{2}+1728 e^{4}-5376 e^{6}+16128 e^{8}\right)\right. \\
& +252\left[11\left(5-54 e^{2}+288 e^{4}-1344 e^{6}\right) \xi^{2}\right. \\
& \left.-\left(35-270 e^{2}+864 e^{4}-1344 e^{6}\right) \zeta^{2}\right] \\
& +1386\left[143\left(1-12 e^{2}+96 e^{4}\right) \xi^{4}\right. \\
& -66\left(5-36 e^{2}+96 e^{4}\right) \zeta^{2} \xi^{2} \\
& \left.+\left(35-180 e^{2}+288 e^{4}\right) \zeta^{4}\right] \\
& +12012\left[143\left(1-18 e^{2}\right) \xi^{6}\right. \\
& +33\left(5-18 e^{2}\right) \xi^{2} \zeta^{4}-429\left(1-6 e^{2}\right) \xi^{4} \zeta^{2} \\
& \left.-\left(7-18 e^{2}\right) \zeta^{6}\right] \\
& +6435\left(2431 \xi^{8}-4004 \zeta^{2} \xi^{6}\right. \\
& \left.\left.+2002 \zeta^{4} \xi^{4}-308 \zeta^{6} \xi^{2}+7 \zeta^{8}\right)\right\} .
\end{aligned}
$$

The terms $\left\langle V_{2}\right\rangle_{u}$ and $\left\langle V_{3}\right\rangle_{u}$ have been repeatedly reported in the literature (Musen 1961; Rosengren \& Scheeres 2013) whereas the remaining terms were verified with corresponding expressions in Delaunay elements that are customarily used in orbit propagators based on semianalytical integration (Lara et al. $2012,2016,2018)$. The constant term in $\left\langle V_{2}\right\rangle_{u}$ is commonly neglected because it has no effect on the long-term motion.

Finally, on account of

$$
\begin{aligned}
& \frac{\partial(\boldsymbol{e} \cdot \boldsymbol{e})}{\partial \boldsymbol{h}}=\mathbf{0}, \\
& \frac{\partial(\boldsymbol{e} \cdot \boldsymbol{e})}{\partial \boldsymbol{e}}=2 \boldsymbol{e}, \\
& \frac{\partial\left(\boldsymbol{e} \cdot \hat{\boldsymbol{r}}_{\star}\right)}{\partial \boldsymbol{h}}=\mathbf{0}, \\
& \frac{\partial\left(\boldsymbol{e} \cdot \hat{\boldsymbol{r}}_{\star}\right)}{\partial \boldsymbol{e}}=\hat{\boldsymbol{r}}_{\star}, \\
& \frac{\partial X}{\partial \boldsymbol{h}}=-2\left(\boldsymbol{h} \cdot \hat{\boldsymbol{r}}_{\star}\right) \hat{\boldsymbol{r}}_{\star}, \\
& \frac{\partial X}{\partial \boldsymbol{e}}=-2 \boldsymbol{e}+2\left(\boldsymbol{e} \cdot \hat{\boldsymbol{r}}_{\star}\right) \hat{\boldsymbol{r}}_{\star},
\end{aligned}
$$

derivation of Eqs. (56)-(57) is straightforward from Eq. (63).

Therefore, using Eq. (25) and taking into account that $(\mu / 2 a) / L=\frac{1}{2} n$, Eqs. (56)-(57) are written in the form

$$
\begin{aligned}
\frac{\mathrm{d} \boldsymbol{h}}{\mathrm{d} t}= & n \epsilon \sum_{i \geq 2} \frac{1}{2^{i}} \frac{a^{i-2}}{r_{\star}^{i-2}}\left[\gamma_{i}\left(\boldsymbol{h} \times \hat{\boldsymbol{r}}_{\star}\right)+\rho_{i}\left(\boldsymbol{e} \times \hat{\boldsymbol{r}}_{\star}\right)\right], \\
\frac{\mathrm{d} \boldsymbol{e}}{\mathrm{d} t}= & n \epsilon \sum_{i \geq 2} \frac{1}{2^{i}} \frac{a^{i-2}}{r_{\star}^{i-2}}\left[\gamma_{i}\left(\boldsymbol{e} \times \hat{\boldsymbol{r}}_{\star}\right)+\rho_{i}\left(\boldsymbol{h} \times \hat{\boldsymbol{r}}_{\star}\right)\right. \\
& \left.+4 \rho_{i-1}(\boldsymbol{h} \times \boldsymbol{e})\right],
\end{aligned}
$$

where we abbreviate $\epsilon=\chi_{\star}\left(n_{\star} / n\right)^{2}\left(a_{\star} / r_{\star}\right)^{3}$. The polynomials $\rho_{i}$ are computed from the recursion

$$
\begin{aligned}
\rho_{i}= & \sum_{l=0}^{\lfloor i / 2\rfloor}\left(\begin{array}{l}
i \\
l
\end{array}\right)\left(\begin{array}{c}
2 i-2 l \\
i
\end{array}\right) \sum_{j=0}^{2 l+1}\left(\begin{array}{c}
2 l+1 \\
j
\end{array}\right) \sum_{k=0}^{i-2 l}\left(\begin{array}{c}
i-2 l \\
k
\end{array}\right) \sum_{m=0}^{k}\left(\begin{array}{l}
k \\
m
\end{array}\right) \sum_{s=0}^{\frac{i-k}{2}-l}\left(\begin{array}{c}
\frac{i-k}{2}-l \\
s
\end{array}\right) \\
& \times \Xi_{j+k-m}^{i-2 l-k}(-1)^{j+l+m+s} e^{j+m-k-2 s} \xi^{2 s+k-1} X^{\frac{i-k}{2}-l-s-1} \\
& \times\left[(k+2 s) X+(i-k-2 l-2 s) \xi^{2}\right],
\end{aligned}
$$

and the polynomials $\gamma_{i}$ from

$$
\begin{aligned}
\gamma_{i}= & \sum_{l=0}^{\lfloor i / 2\rfloor}\left(\begin{array}{c}
i \\
l
\end{array}\right)\left(\begin{array}{c}
2 i-2 l \\
i
\end{array}\right) \sum_{j=0}^{2 l+1}\left(\begin{array}{c}
2 l+1 \\
j
\end{array}\right) \sum_{k=0}^{i-2 l}\left(\begin{array}{c}
i-2 l \\
k
\end{array}\right) \sum_{m=0}^{k}\left(\begin{array}{c}
k \\
m
\end{array}\right) \sum_{s=0}^{\frac{i-k}{2}-l}\left(\begin{array}{c}
\frac{i-k}{2}-l \\
s
\end{array}\right) \\
& \times \Xi_{j+k-m}^{i-2 l-k}(-1)^{j+l+m+s+1}(i-k-l-s) e^{j+m-k-2 s} \xi^{2 s+k} \zeta X^{\frac{i-k}{2}-l-s-1} .
\end{aligned}
$$

The complexity of the polynomials given by Eqs. (79) and (80) is apparent, and corresponding expressions take a compact form, which is illustrated in Table 1 for the lower degrees.

\section{Performance evaluation: The case of high Earth orbits}

The simplicity of the vectorial approach in approximating the long-term dynamics of a system under third-body perturbations when compared with classical expansions based on trigonometric terms is evident from the visual comparison of Table 1 with equivalent results based on classical trigonometric expansions. For instance, while the entire Tables 4-8 of Lara et al. (2018) are required to evaluate the third-body disturbing acceleration up to degree 6 of the Legendre polynomial expansion, which calls for the additional evaluation of trigonometric functions, only the upper half of Table 1 is required when the vectorial approach is 
Table 1. Some polynomials $\rho_{i}, \gamma_{i}$, given by Eqs. (79)-(80).

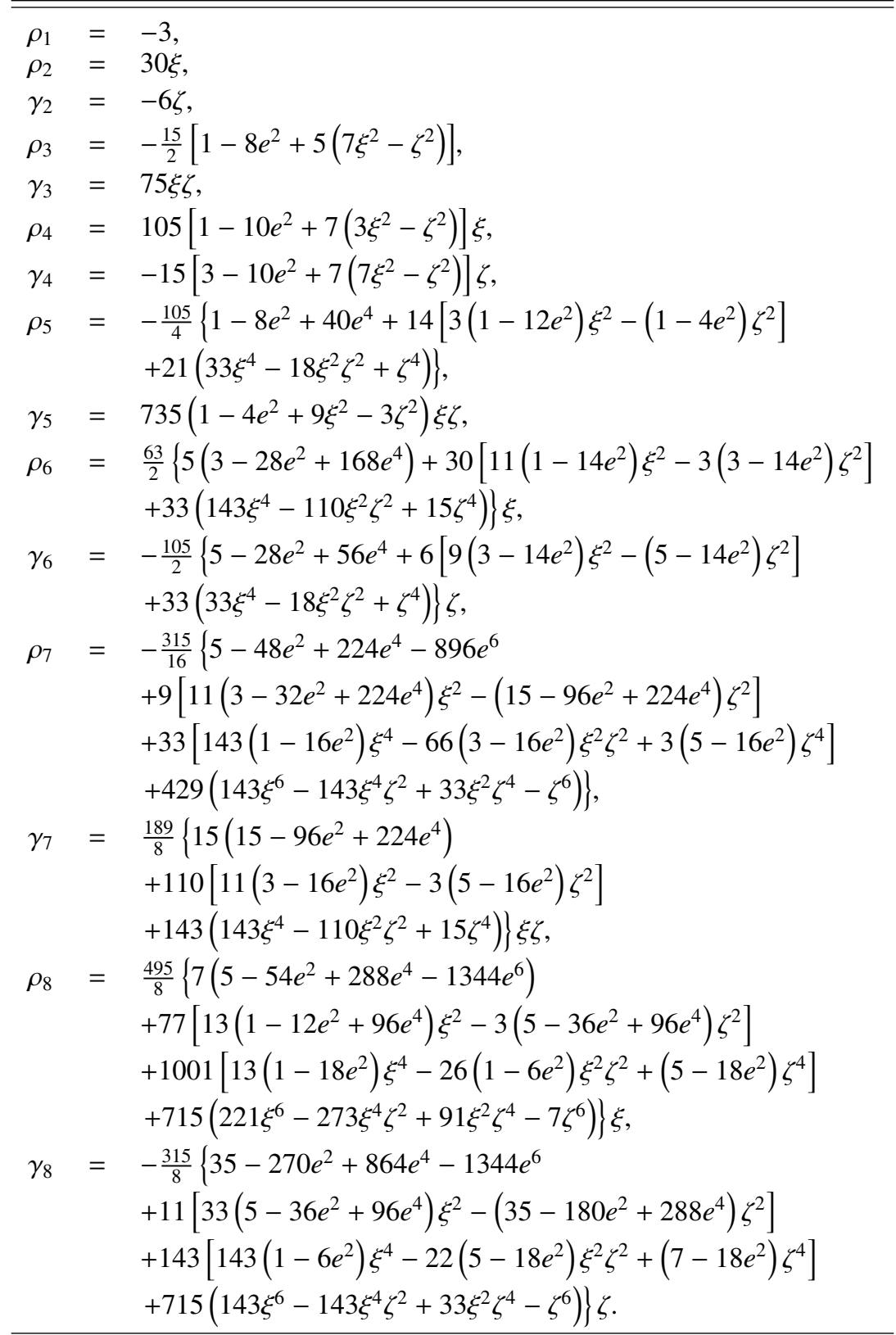

used. On the other hand, the numerical integration of the mean elements is expected to progress with similar step sizes when either vectorial or other classical mean elements are used. The former requires integrating a redundant differential system of higher dimension, however, which might counterbalance the presumed advantages derived from the simplicity of the formulation and the use of arithmetic operations only, as opposed to evaluating trigonometric functions.

The improvements obtained with the vectorial formulation when higher degrees are required in expanding the third-body disturbing function are illustrated for high Earth orbits. In particular, we present an example of a SIMBOL-X-type orbit, whose semianalytical propagation requires the use of at least a $P_{2}-P_{6}$ truncation of the lunar disturbing function in order to capture the main frequencies of the long-term motion (Lara et al. 2018; Amato et al. 2019).
For our efficiency proofs, we assume the initial conditions

$$
\begin{aligned}
& a=106247.136 \mathrm{~km} \\
& e=0.75173 \\
& I=5.2789^{\circ} \\
& \Omega=49.351^{\circ} \\
& \omega=180.008^{\circ} \\
& M=0
\end{aligned}
$$

where $a, e, I, \Omega, \omega$, and $M$ stand for classical Keplerian elements, and remove all perturbations except for the lunisolar ones. The initial epoch needed for the computation of the lunisolar ephemeris is fixed to 2014 July 1 at $20.7208333 \mathrm{~h}$ UTC. Then, we propagate these initial conditions for intervals of 100 years with both the vectorial approach of this paper and with the classical approach used by Lara et al. (2018). The solar effect is truncated to the $P_{2}$ Legendre polynomial whereas lunar perturbations 


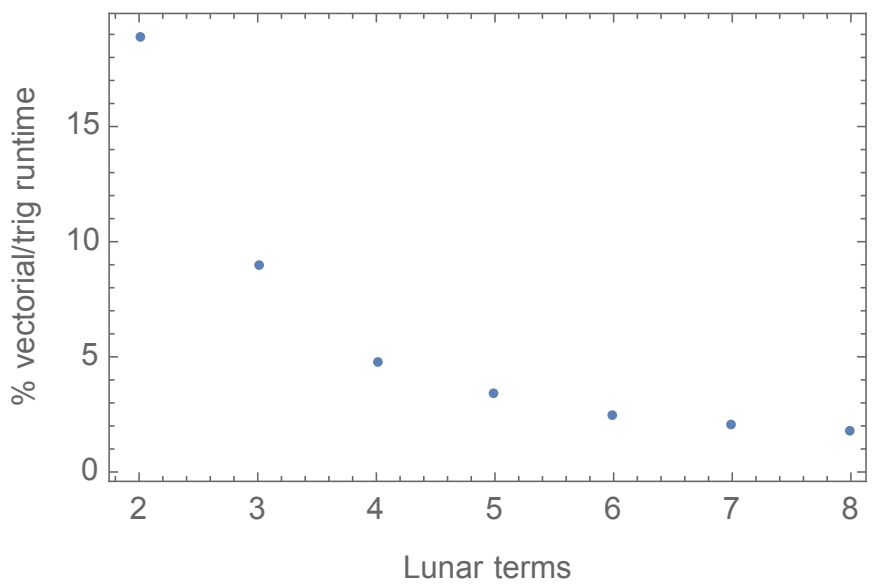

Fig. 1. Runtime of the vectorial approach relative to the classical trigonometric implementation in percentage for an increasing complexity of the lunar perturbation.

only include from $P_{2}$ to $P_{2}-P_{8}$ in successive increments of one term. The numerical integration of the averaged flow is carried out with the reputed DOPRI 853 integrator, which implements an explicit Runge-Kutta method of order $8(5,3)$ due to Dormand \& Prince (1980), with step size control and dense output (Hairer et al. 2008).

In order to estimate the gains of the vectorial formulation with respect to the trigonometric expansions, we first make the propagations with constant step size and without updating the lunisolar ephemerides. The results are depicted in Fig. 1 in terms of the runtime percentage of the vectorial approach relative to the classical propagation. Because the figures we present are relative quantities, analogous results are expected using different computational environments and therefore, no additional information on the hardware and software used in the tests is needed. However, we did not optimize the evaluation of the disturbing acceleration, and except for trivial arrangements, we completely left the optimization task to the compiler in both cases: in the vectorial formulation of this paper, and in the classical approach of Lara et al. (2018).

Figure 1 shows that when the lunar perturbation is limited to the quadrupolar term, the vectorial formulation only takes about one fifth of the time required by the classical formulation in trigonometric functions to complete the 100-year propagation. The gains of the vectorial formulation notably increase with the fidelity in modeling lunar perturbations. The vectorial approach performs in less than one tenth of the classical approach of Lara et al. (2018) when the lunar octupolar term is also taken into account, and takes an impressive $1.8 \%$ of the time required by the classical formulation when the terms $P_{2}-P_{8}$ are taken into account. We recall that for this particular orbit, the long-term dynamics is correctly modeled with a $P_{2}-P_{6}$ truncation of the lunar disturbing function, cf. Lara et al. (2018), a case in which the runtime obtained with the vectorial formulation is shorter than $2.5 \%$ of the time required by the trigonometric expansions.

On the other hand, the evaluation of the third-body acceleration, as given by Eqs. (77)-(78) or equivalent expressions in Lara et al. (2018), is only a part of the computational burden of the propagation. Evaluating the disturbing acceleration requires the previous knowledge of the third-body ephemeris at each step of the numerical integration of the flow in mean elements. While this task may be computationally undemanding for simple restricted three-body models in which the orbit of the

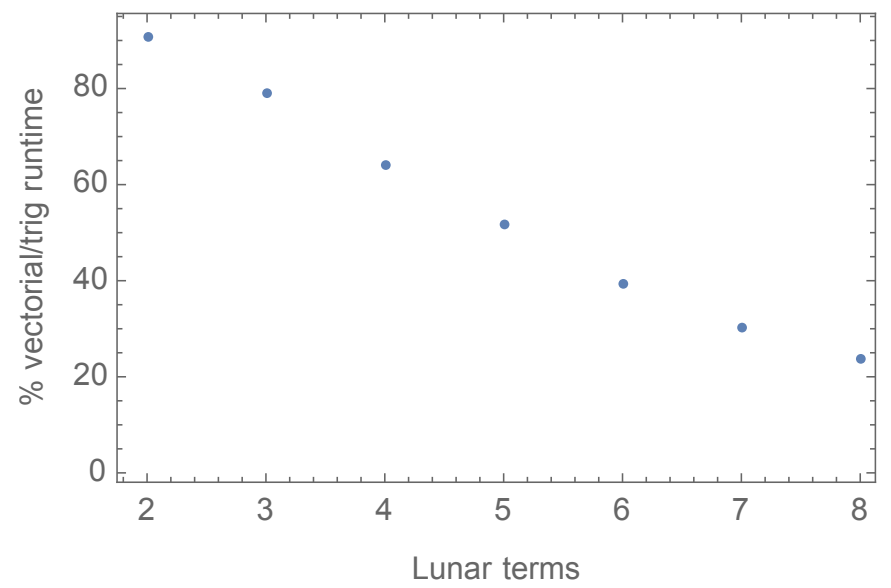

Fig. 2. Same as Fig. 1 when the time spent to evaluate the lunisolar ephemerides is included.

disturber may be taken as purely Keplerian (Lara et al. 2012), that is not at all the case for Earth-orbiting satellites, in which lunar and solar ephemerides must either be read from a data file or be evaluated from analytical expressions. In this last case, the added computational effort is not at all negligible, and the question therefore naturally emerges whether the efforts in improving the evaluation of the differential equations of the flow might be vacuous. To verify that, we repeat the computations in the actual case in which lunisolar ephemerides are computed at each integration step of the numerical integration procedure. Corresponding results are shown in Fig. 2. Runtime improvements are quite small for the quadrupolar case and only moderate for the octupolar case. The vectorial approach still halves the runtime when moderate degrees are required in the expansion of the lunar disturbing function, and only about $20 \%$ of the runtime of the classical approach is required when the truncation is extended to the $P_{2}-P_{8}$ case.

Finally, we recall that an added bonus on the side of the vectorial formulation is that its redundancy allows us to examine the accuracy of the numerical integration by evaluating the geometrical relations $\boldsymbol{h} \cdot \boldsymbol{e}=0$ and $\boldsymbol{e} \cdot \boldsymbol{e}+\boldsymbol{h} \cdot \boldsymbol{h}=1$, cf. Herrick (1948). Using these tests, we found that the errors of the 100 -year numerical propagation of the flow in mean vectorial elements is constrained to the order of $10^{-14}$ when the tolerance of the numerical integrator is set to $10^{-14}$.

\section{Conclusions}

We have provided a detailed description of the Hamiltonian reduction process that removes short-period effects from the third-body disturbing function, keeping the slowly evolving parameters in vectorial form. Our approach takes advantage of the apsidal frame formulation to provide general expressions of the mean element potential in vectorial form up to an arbitrary degree. The variation of parameter equations of the flow in mean elements admits a general, nonsingular, compact formulation when the elements are the angular momentum vector and the eccentricity vector. We verified that the explicit vectorial expressions for the lower degree truncations of our general approach agree with alternative derivations in the literature, and they can now be extended to an arbitrary degree. In addition, the compact form of the vectorial expressions permits avoiding the long listings required when angular variables are used, and printed expressions up to moderate orders of the variation equations 
can be arranged in only one side of a letter. Runs for particular examples confirmed the expected notable improvements of the performance in the propagation of the mean elements' flow when the vectorial approach was used.

Acknowledgements. The work of ML and EF has been funded by Khalifa University of Science and Technology's internal grants FSU-2018-07 and CIRA2018-85. ML also thanks partial support by the Spanish State Research Agency and the European Regional Development Fund under Projects ESP2016-76585$\mathrm{R}$ and ESP2017-87271-P (AEI/ ERDF, EU).

\section{References}

Allan, R. R. 1962, Q. J. Mech. Appl. Math., 15, 283

Allan, R. R., \& Cook, G. E. 1964, Proc. R. Soc. London Ser. A, 280, 97

Allan, R. R., \& Ward, G. N. 1963, Proc. Camb. Philos. Soc., 59, 669

Amato, D., Bombardelli, C., Baú, G., Morand, V., \& Rosengren, A. J. 2019, Celest. Mech. Dyn. Astron., 131, 21

Andrade-Ines, E., \& Robutel, P. 2018, Celest. Mech. Dyn. Astron., 130, 6

Andrade-Ines, E., Beaugé, C., Michtchenko, T., \& Robutel, P. 2016, Celest. Mech. Dyn. Astron., 124, 405

Beaugé, C., \& Michtchenko, T. A. 2003, MNRAS, 341, 760

Blanes, S., Casas, F., Farrés, A., et al. 2013, Appl. Numer. Math., 68 58

Boccaletti, D., \& Pucacco, G. 2002, in Theory of Orbits. Volume 2: Perturbative and Geometrical Methods, 1st edn. (Berlin, Heidelberg, New York: SpringerVerlag), Astron. Astrophys. Lib.

Breiter, S., \& Ratajczak, R. 2005, MNRAS, 364, 1222

Celletti, A., Galeş, C., Pucacco, G., \& Rosengren, A. J. 2017, Celest. Mech. Dyn. Astron., 127, 259

Correia, A. C. M., Laskar, J., Farago, F., \& Boué, G. 2011, Celest. Mech. Dyn. Astron., 111, 105

Deprit, A. 1969, Celest. Mech., 1, 12

Deprit, A. 1975, J. Res. Natl. Bur. Stand., 79, 1

Deprit, A. 1982, Celest. Mech., 26, 9

Deprit, A. 1983, Celest. Mech., 29, 229

Deprit, A. 1984, in The Big-Bang and Georges Lemaître, ed. A. Berger (Dordrecht: Springer), 151

Dormand, J. R., \& Prince, P. J. 1980, J. Comput. Appl. Math., 6, 19

Giacaglia, G. E. O. 1974, Celest. Mech., 9, 239

Hairer, E., Nørset, S. P., \& Wanner, G. 2008, Solving Ordinary Differential Equations I. Non-stiff Problems, 2nd edn. (Berlin, Heidelberg, New York Springer-Verlag)

Hamers, A. S., Perets, H. B., Antonini, F., \& Portegies Zwart, S. F. 2015 MNRAS, 449, 4221
Hansen, P. A. 1855, Abhandlungen der Koniglich Sachsischen Gesellschaft der Wissenschaften, 2, 183, English translation by J. C. Van der Ha, ESA/ESOC, Darmstadt, Germany, 1977

Hansen, P. A. 1857, Abhandlungen der Koniglich Sachsischen Gesellschaft der Wissenschaften, 5, 41

Henrard, J. 1974, Celest. Mech., 10, 437

Herrick, S. 1948, PASP, 60, 321

Hintz, G. 2008, J. Guid. Control Dyn., 31, 785

Hori, G.-I. 1966, PASJ, 18, 287

Katz, B., Dong, S., \& Malhotra, R. 2011, Phys. Rev. Lett., 107, 181101

Kaula, W. M. 1962, AJ, 67, 300

Kaula, W. M. 1966, Theory of Satellite Geodesy. Applications of Satellites to Geodesy (Waltham, Massachusetts: Blaisdell)

Kelly, T. S. 1989, Celest. Mech. Dyn. Astron., 46, 19

Lane, M. T. 1989, Celest. Mech. Dyn. Astron., 46, 287

Lara, M. 2016, in Astrodynamics Network AstroNet-II: The Final Conference, eds. G. Gómez, \& J. Masdemont (Springer: Cham), Astrophys. Space Sci. Proc., 44, 151

Lara, M. 2017, Celest. Mech. Dyn. Astron., 129, 137

Lara, M., San-Juan, J. F., López, L. M., \& Cefola, P. J. 2012, Celest. Mech. Dyn. Astron., 113, 435

Lara, M., San-Juan, J., \& Hautesserres, D. 2016, Semi-analytical Propagator of High Eccentricity Orbits, Technical Report R-S15/BS-0005-024, Centre Nationald'Études Spatiales, 18, Avenue Edouard Belin, 31401, Toulouse Cedex 9, France

Lara, M., San-Juan, J. F., \& Hautesserres, D. 2018, CEAS Space J., 10, 3

Laskar, J., \& Boué, G. 2010, A\&A, 522, A60

Laskar, J., \& Robutel, P. 2001, Celest. Mech. Dyn. Astron., 80, 39

Lee, M. H., \& Peale, S. J. 2003, ApJ, 592, 1201

Libert, A. S., \& Sansottera, M. 2013, Celest. Mech. Dyn. Astron., 117, 149

Mardling, R. A. 2013, MNRAS, 435, 2187

Meyer, K. R., \& Hall, G. R. 1992, Introduction to Hamiltonian Dynamical Systems and the N-Body Problem (New York: Springer)

Migaszewski, C., \& Goździewski, K. 2008, MNRAS, 388, 789

Mignard, F., \& Henon, M. 1984, Celest. Mech., 33, 239

Milankovitch, M. 1941, Kanon der Erdbestrahlung und seine Anwendung auf das Eiszeitenproblem, Mechanics of Space Flight (Belgrade: Königlich Serbische Akademie), English translation: Canon of Insolation and the Ice-age Problem. Israel Program for Scientific Translations, Jerusalem, 1969

Musen, P. 1961, J. Geophys. Res., 66, 2797

Musen, P. 1963, J. Geophys. Res., 68, 6255

Palacián, J. F., Vanegas, J., \& Yanguas, P. 2017, Astrophys. Space Sci., 362, 215 Richter, K., \& Keller, H. U. 1995, Icarus, 114, 355

Rosengren, A., \& Scheeres, D. 2013, Adv. Space Res., 52, 1545

Rosengren, A. J., \& Scheeres, D. J. 2014, Celest. Mech. Dyn. Astron., 118, 197

Roy, A. E., \& Moran, P. E. 1973, Celest. Mech., 7, 236

Sansottera, M., \& Libert, A. S. 2019, Celest. Mech. Dyn. Astron., 131, 38

Will, C. M. 2017, Phys. Rev. D, 96, 023017 